\title{
In silico structural modelling and analysis of Elongation factor-1 alpha and Elongation factor-like protein
}

\section{Supporting Information}

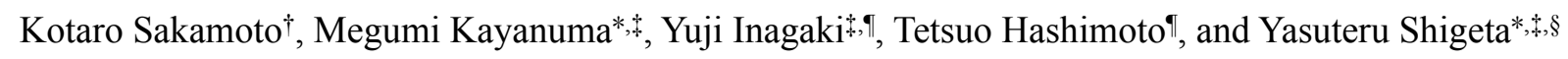

'Leading Graduate School Doctoral Program in Human Biology, University of Tsukuba, Ibaraki 305-8577, Japan.

†enter for Computational Sciences, University of Tsukuba, Ibaraki 305-8577, Japan.

'Graduate School of Life and Environmental Sciences, University of Tsukuba, Ibaraki305-8577, Japan.

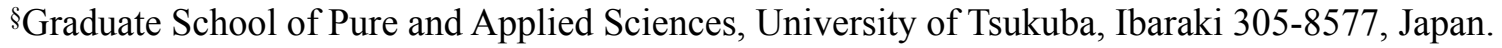

E-mail:kayanuma@ccs.tsukuba.ac.jp; shigeta@ccs.tsukuba.ac.jp 
Figure S1

Crystal structures of the template and the molecular surfaces taken from eF-site [33] of yeast eEF1A (PDB ID: 1G7C (A) and eF-site ID: 1g7c-A (B)), rabbit eEF1a (PDB ID: 4C0S (C) and eF-site ID: 4c0s-A (D)), and Aeropyrum pernix aEF1a (PDB ID: 3WXM (E) and eF-site ID: 3wxm-A (F)).

The aa-tRNA binding sites are indicated in yellow colour on the ribbon representations of the template proteins (Left)
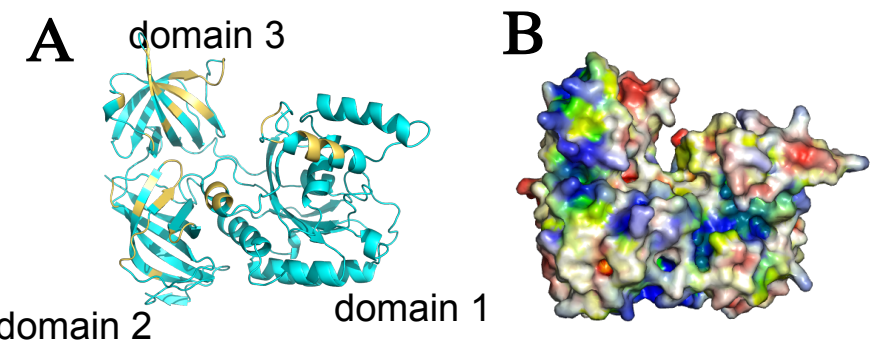

C

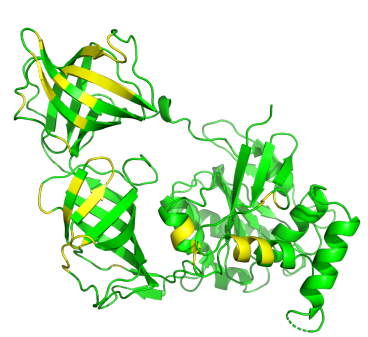

$\mathbf{E}$

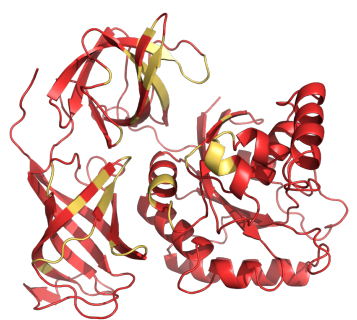

D

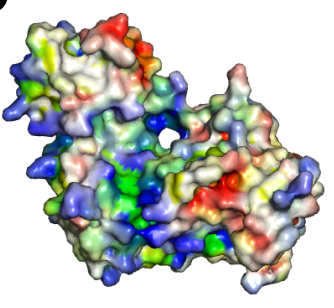

$\mathbf{F}$

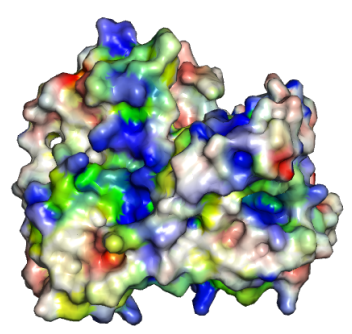

electrostatic potential

$-0.1 \mathrm{~V}$

$+0.1 \mathrm{~V}$

hydrophilic

hydrophobic 
Table S1

Homology models of EF-1 $\alpha$ and EFL proteins built by SWISS-MODEL web server [30] using 1G7C in PDB as the template.

\begin{tabular}{|c|c|c|c|c|}
\hline protein & taxon name & Identity & GIVIQE & QIVIEAN \\
\hline \multirow{10}{*}{$\begin{array}{c}E F-1 \alpha \text { of } \\
\text { EF-1 } 1 \alpha-c o n t a i n i n g \\
\text { species }\end{array}$} & Bolidomonas pacifica CCMP 1866 & 77.10 & 0.93 & -0.05 \\
\hline & Goniomonas sp. ATCC PRA68 & 78.73 & 0.88 & -0.01 \\
\hline & Corallochytrium limacisporum AY582826 & 81.46 & 0.93 & 0.41 \\
\hline & Goniomonas truncata NIES 1373 & 79.06 & 0.90 & -0.03 \\
\hline & Goniomonas sp. CCAP 980_1 & 77.30 & 0.84 & 0.03 \\
\hline & Subulatomonas sp. strain PCMinv5 & 75.29 & 0.90 & 0.15 \\
\hline & Breviata-like biflagellate strain PCbi66 & 80.14 & 0.88 & 0.38 \\
\hline & Roombia sp. NY0200 & 77.01 & 0.84 & -0.40 \\
\hline & Mantamonas plastica Bass1 (CCAP 1946/1) & 79.30 & 0.87 & 0.30 \\
\hline & microaerophilic cercozoan strain DMV & 70.56 & 0.78 & -0.48 \\
\hline \multirow{5}{*}{$\begin{array}{c}\text { EFL of } \\
\text { EFL-containing species }\end{array}$} & Goniomonas sp. NIES 1374 & 36.60 & 0.69 & -2.81 \\
\hline & Raphidiophrys contractilis AB332032 & 45.73 & 0.73 & -3.00 \\
\hline & Planoglabratella opercularis EU810334 & 42.42 & 0.71 & -2.22 \\
\hline & Capromyxa protea CF08-5 (ATCC PRA-324) & 42.27 & 0.70 & -2.48 \\
\hline & Fabomonas tropica strain NYK3C & 43.96 & 0.73 & -3.00 \\
\hline \multirow{6}{*}{$\begin{array}{c}\text { EFL of } \\
\text { dual-EF-containing } \\
\text { species }\end{array}$} & Achnanthes kuwaitensis NIES1349 & 41.67 & 0.74 & -1.01 \\
\hline & Asterionella glacialis NIES417 & 42.11 & 0.74 & -1.32 \\
\hline & Detonula confervcace CCMP353 & 43.23 & 0.76 & -1.18 \\
\hline & Spizellomyces punctatus DAOM BR117 & 41.72 & 0.65 & -3.15 \\
\hline & Thalassionema nitzschioides NIES534 & 43.30 & 0.75 & -1.14 \\
\hline & Thecamonas trahens ATCC50062 & 44.55 & 0.70 & -3.33 \\
\hline \multirow{10}{*}{$\begin{array}{l}\text { Divergent EF-1 } \alpha \text { of } \\
\text { dual-EF-containing } \\
\text { species }\end{array}$} & Achnanthes kuwaitensis NIES1349 & 66.83 & 0.81 & 0.03 \\
\hline & Asterionella glacialis NIES417 & 70.17 & 0.85 & -0.46 \\
\hline & Detonula confervacea CCMP353 & 65.77 & 0.83 & -0.72 \\
\hline & Goniomonas sp. ATCC 50180 & 63.51 & 0.78 & -0.22 \\
\hline & Fragilariopsis cylindrus & 53.57 & 0.77 & -1.01 \\
\hline & Pythium intermedium MAFF306022 & 59.18 & 0.77 & -0.13 \\
\hline & Pythium ultimum DAOM BR144 & 50.35 & 0.79 & -0.73 \\
\hline & Spizellomyces punctatus DAOM BR117 & 60.41 & 0.78 & -1.35 \\
\hline & Thalassionema nitzschioides NIES534 & 58.70 & 0.78 & -0.83 \\
\hline & Thecamonas trahens ATCC50062 & 68.20 & 0.76 & -0.27 \\
\hline
\end{tabular}


Table S2

Homology models of EF-1 $\alpha$ and EFL proteins built by SWISS-MODEL web server [30] using 4C0S in PDB as the template.

\begin{tabular}{|c|c|c|c|c|}
\hline protein & taxon name & Identity & GIVIQE & QIVIEAN \\
\hline \multirow{10}{*}{$\begin{array}{c}E F-1 \alpha \text { of } \\
\text { EF-1 } 1 \alpha-c o n t a i n i n g \\
\text { species }\end{array}$} & Bolidomonas pacifica CCMP 1866 & 75.70 & 0.90 & -1.27 \\
\hline & Goniomonas sp. ATCC PRA68 & 79.27 & 0.85 & -1.70 \\
\hline & Corallochytrium limacisporum AY582826 & 82.30 & 0.96 & -0.71 \\
\hline & Goniomonas truncata NIES 1373 & 79.90 & 0.89 & -1.46 \\
\hline & Goniomonas sp. CCAP 980_1 & 77.23 & 0.84 & -2.33 \\
\hline & Subulatomonas sp. strain PC Minv5 & 76.78 & 0.91 & -1.00 \\
\hline & Breviata-like biflagellate strain PCbi66 & 81.31 & 0.88 & -1.75 \\
\hline & Roombia sp. NY0200 & 78.67 & 0.85 & -1.92 \\
\hline & Mantamonas plastica Bass1 (CCAP 1946/1) & 81.86 & 0.87 & -1.12 \\
\hline & microaerophilic cercozoan strain DMV & 70.82 & 0.80 & -1.61 \\
\hline \multirow{5}{*}{$\begin{array}{c}\text { EFL of } \\
\text { EFL-containing species }\end{array}$} & Goniomonas sp. NIES 1374 & 37.32 & 0.69 & -4.39 \\
\hline & Raphidiophrys contractilis AB332032 & 46.84 & 0.75 & -2.72 \\
\hline & Planoglabratella opercularis EU810334 & 44.78 & 0.72 & -2.65 \\
\hline & Capromyxa protea CF08-5 (ATCC PRA-324) & 42.31 & 0.71 & -3.19 \\
\hline & Fabomonas tropica strain NYK3C & 46.28 & 0.75 & -2.58 \\
\hline \multirow{6}{*}{$\begin{array}{c}\text { EFL of } \\
\text { dual-EF-containing } \\
\text { species }\end{array}$} & Achnanthes kuwaitensis NIES1349 & 41.90 & 0.74 & -0.81 \\
\hline & Asterionella glacialis NIES417 & 42.82 & 0.75 & -1.12 \\
\hline & Detonula confervcace CCMP353 & 43.23 & 0.76 & -1.18 \\
\hline & Spizellomyces punctatus DAOM BR117 & 45.18 & 0.68 & -2.51 \\
\hline & Thalassionema nitzschioides NIES534 & 43.30 & 0.75 & -1.14 \\
\hline & Thecamonas trahens ATCC50062 & 44.34 & 0.70 & -3.30 \\
\hline \multirow{10}{*}{$\begin{array}{l}\text { Divergent EF-1 } \alpha \text { of } \\
\text { dual-EF-containing } \\
\text { species }\end{array}$} & Achnanthes kuwaitensis NIES1349 & 68.05 & 0.81 & -1.08 \\
\hline & Asterionella glacialis NIES417 & 68.54 & 0.78 & -1.87 \\
\hline & Detonula confervacea CCMP353 & 67.80 & 0.78 & -1.81 \\
\hline & Goniomonas sp. ATCC 50180 & 65.48 & 0.78 & -2.10 \\
\hline & Fragilariopsis cylindrus & 57.01 & 0.76 & -1.86 \\
\hline & Pythium intermedium MAFF306022 & 61.30 & 0.77 & -1.20 \\
\hline & Pythium ultimum DAOM BR144 & 50.81 & 0.77 & -0.90 \\
\hline & Spizellomyces punctatus DAOM BR117 & 60.23 & 0.82 & -1.35 \\
\hline & Thalassionema nitzschioides NIES534 & 60.34 & 0.78 & -1.55 \\
\hline & Thecamonas trahens ATCC50062 & 67.05 & 0.76 & -1.71 \\
\hline
\end{tabular}


Table S3

Homology models of EF-1 $\alpha$ and EFL proteins built by SWISS-MODEL web server [30] using 3WXM in PDB as the template.

\begin{tabular}{|c|c|c|c|c|}
\hline protein & taxon name & Identity & GIVIQE & QIVIEAN \\
\hline \multirow{10}{*}{$\begin{array}{c}E F-1 \alpha \text { of } \\
\text { EF-1 } 1 \alpha-c o n t a i n i n g \\
\text { species }\end{array}$} & Bolidomonas pacifica CCMP 1866 & 55.73 & 0.80 & -1.11 \\
\hline & Goniomonas sp. ATCC PRA68 & 51.46 & 0.72 & -1.72 \\
\hline & Corallochytrium limacisporum AY582826 & 55.77 & 0.79 & -1.36 \\
\hline & Goniomonas truncata NIES 1373 & 51.23 & 0.78 & -1.37 \\
\hline & Goniomonas sp. CCAP 980_1 & 51.71 & 0.74 & -1.61 \\
\hline & Subulatomonas sp. strain PCMinv5 & 53.23 & 0.80 & -1.29 \\
\hline & Breviata-like biflagellate strain PCbi66 & 55.71 & 0.76 & -0.96 \\
\hline & Roombia sp. NY0200 & 52.93 & 0.76 & -1.03 \\
\hline & Mantamonas plastica Bass1 (CCAP 1946/1) & 56.31 & 0.76 & -1.29 \\
\hline & microaerophilic cercozoan strain DMV & 54.99 & 0.76 & -0.94 \\
\hline \multirow{5}{*}{$\begin{array}{c}\text { EFL of } \\
\text { EFL-containing species }\end{array}$} & Goniomonas sp. NIES 1374 & 42.86 & 0.69 & -2.97 \\
\hline & Raphidiophrys contractilis AB332032 & 43.14 & 0.71 & -2.80 \\
\hline & Planoglabratella opercularis EU810334 & 43.84 & 0.69 & -3.51 \\
\hline & Capromyxa protea CF08-5 (ATCC PRA-324) & 44.02 & 0.68 & -4.11 \\
\hline & Fabomonas tropica strain NYK3C & 40.52 & 0.70 & -2.96 \\
\hline \multirow{6}{*}{$\begin{array}{c}\text { EFL of } \\
\text { dual-EF-containing } \\
\text { species }\end{array}$} & Achnanthes kuwaitensis NIES1349 & 41.18 & 0.72 & -1.11 \\
\hline & Asterionella glacialis NIES417 & 41.98 & 0.70 & -1.85 \\
\hline & Detonula confervcace CCMP353 & 41.77 & 0.71 & -1.41 \\
\hline & Spizellomyces punctatus DAOM BR117 & 41.65 & 0.64 & -3.03 \\
\hline & Thalassionema nitzschioides NIES534 & 42.93 & 0.73 & -0.76 \\
\hline & Thecamonas trahens ATCC50062 & 42.55 & 0.68 & -3.82 \\
\hline \multirow{10}{*}{$\begin{array}{l}\text { Divergent EF-1a of } \\
\text { dual-EF-containing } \\
\text { species }\end{array}$} & Achnanthes kuwaitensis NIES1349 & 54.28 & 0.77 & -0.98 \\
\hline & Asterionella glacialis NIES417 & 41.98 & 0.70 & -1.85 \\
\hline & Detonula confervacea CCMP353 & 53.64 & 0.76 & -1.19 \\
\hline & Goniomonas sp. ATCC 50180 & 48.18 & 0.75 & -1.46 \\
\hline & Fragilariopsis cylindrus & 45.18 & 0.74 & -1.62 \\
\hline & Pythium intermedium MAFF306022 & 49.64 & 0.77 & -1.66 \\
\hline & Pythium ultimum DAOM BR144 & 47.89 & 0.78 & -1.03 \\
\hline & Spizellomyces punctatus DAOM BR117 & 51.86 & 0.77 & -1.59 \\
\hline & Thalassionema nitzschioides NIES534 & 48.91 & 0.76 & -0.86 \\
\hline & Thecamonas trahens ATCC50062 & 52.73 & 0.75 & -1.09 \\
\hline
\end{tabular}


Figure S2

Molecular surface seen from the tRNA-binding side of EF-1 $\alpha$ and EFL models, generated using SWISS-MODEL using 1G7C in PDB as the template, obtained using eF-surf web server [34]: EF-1a of EF-1a-containing species (Bolidomonas pacifica CCMP 1866 (A), Goniomonas sp. ATCC PRA68 (B), Corallochytrium limacisporum (AY582826) (C), Goniomonas truncata NIES 1373 (D), Goniomonas sp. CCAP 980_1 (E), Subulatomonas sp. strain PCMinv5 (F), Breviata-like biflagellate strain PCbi66 (G), Roombia sp. NY0200 (H), Mantamonas plastica Bass1 (CCAP 1946/1) (I), and microaerophilic cercozoan strain DMV (J)). Divergent EF-1a of dual-EF-containing species (Detonula confervcace CCMP353 (K), Fragilariopsis cylindrus (L), Thalassionema nitzschioides NIES534 (M), Pythium intermedium MAFF306022 (N), Goniomonas sp. ATCC 50180 (O), Achnanthes kuwaitensis NIES1349 (P), Asterionella glacialis NIES417 Q), Pythium ultimum DAOM BR144 (T), Spizellomyces punctatus DAOM BR117 (S), and Thecamonas trahens ATCC50062 (T)). EFL of dual-EF-containing species (Achnanthes kuwaitensis NIES1349 (U), Asterionella glacialis NIES417 (V), Spizellomyces punctatus DAOM BR117 (W) and Thecamonas trahens ATCC50062(X), Detonula confervcace CCMP353 (Y), Thalassionema nitzschioides NIES534 (Z), EFL of EFL-containing species (Goniomonas sp. NIES 1374 (A'), Raphidiophrys contractilis (AB332032) (B'), Planoglabratella opercularis (EU810334) (C'), Capromyxa protea CF08-5 (ATCC PRA-324) (D'), and Fabomonas tropica strain NYK3C (E')).
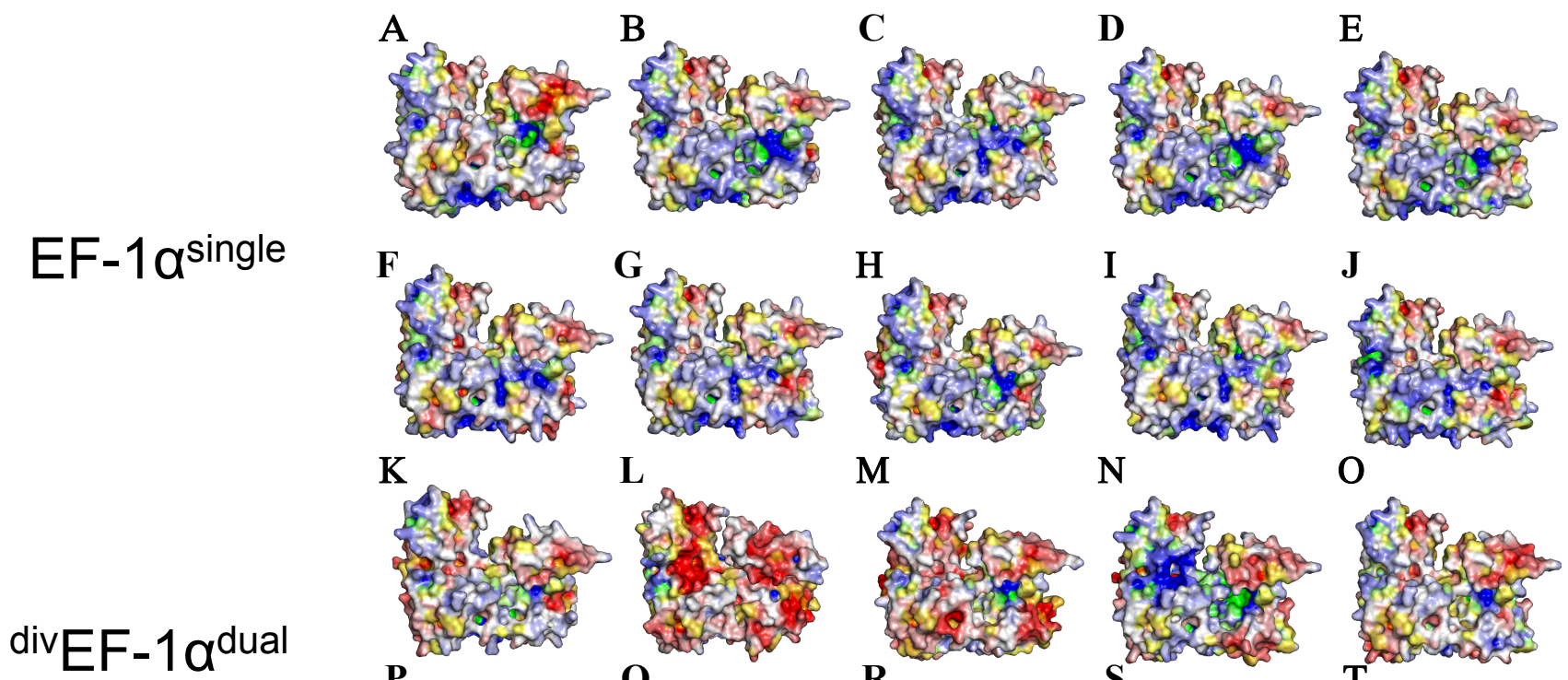

$\mathrm{K}$
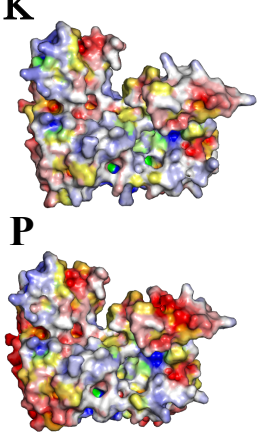

$\mathbf{L}$
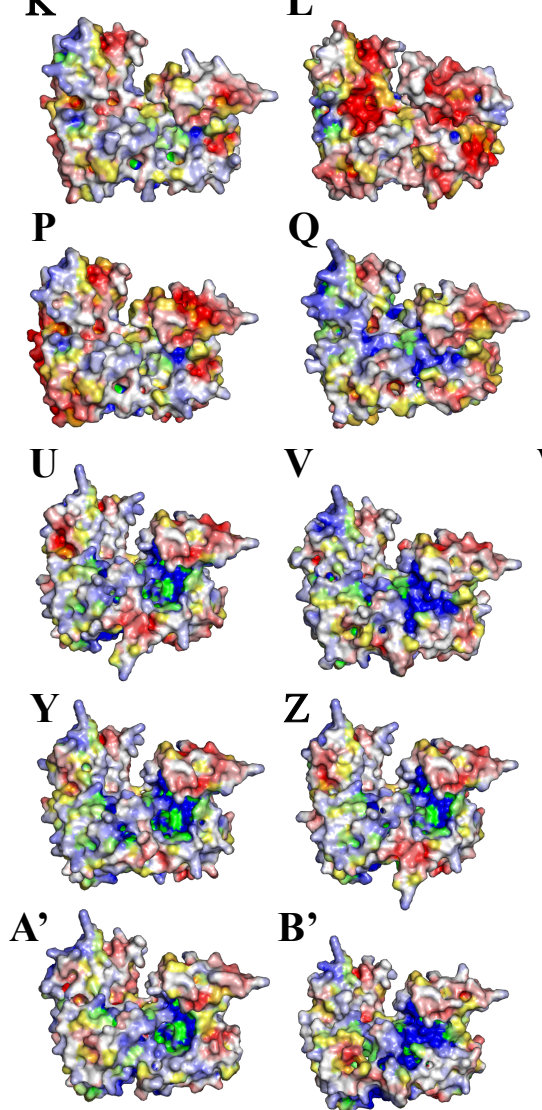
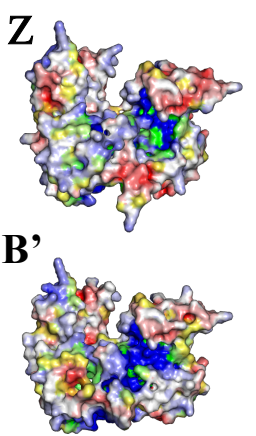
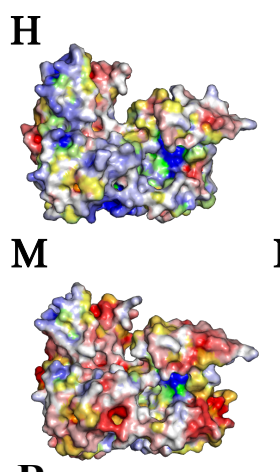

R

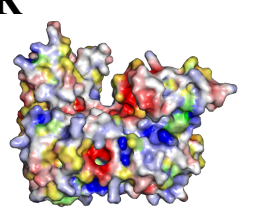

W
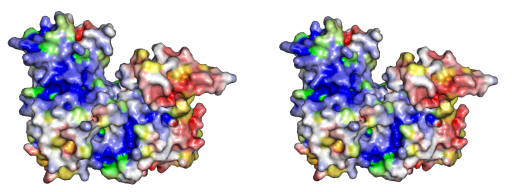

I

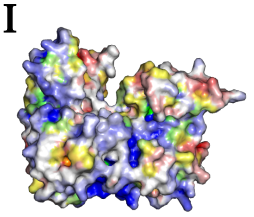

$\mathbf{N}$

J

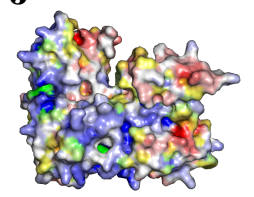

0

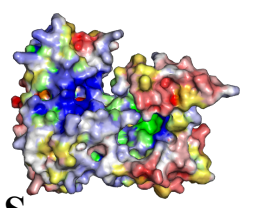

S

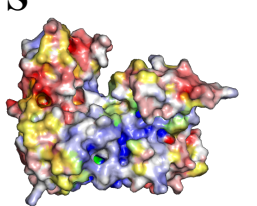

$\mathbf{X}$

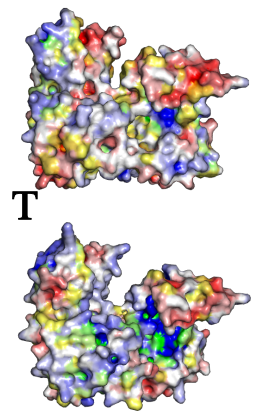

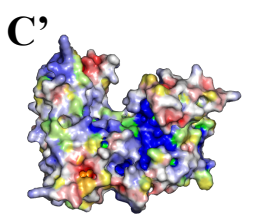

S6

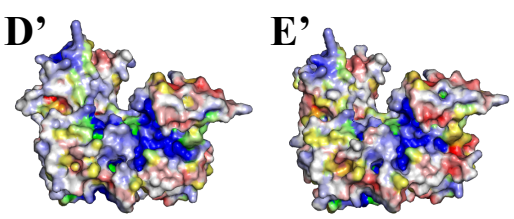

electrostatic potential $+0.1 \mathrm{~V}$

$-0.1 \mathrm{~V}$ hydrophilic hydrophobic 
Figure S3

Molecular surface seen from the tRNA-binding side of EF-1a and EFL models, generated using SWISS-MODEL using 4COS in PDB as the template, obtained using eF-surf web server [34]: EF-1a of EF-1a-containing species (Bolidomonas pacifica CCMP 1866 (A), Goniomonas sp. ATCC PRA68 (B), Corallochytrium limacisporum (AY582826) (C), Goniomonas truncata NIES 1373 (D), Goniomonas sp. CCAP 980_1 (E), Subulatomonas sp. strain PCMinv5 (F), Breviata-like biflagellate strain PCbi66 (G), Roombia sp. NY0200 (H), Mantamonas plastica Bass1 (CCAP 1946/1) (I), and microaerophilic cercozoan strain DMV (J)). Divergent EF-1a of dual-EF-containing species (Detonula confervcace CCMP353 (K), Fragilariopsis cylindrus (L), Thalassionema nitzschioides NIES534 (M), Pythium intermedium MAFF306022 (N), Goniomonas sp. ATCC 50180 (O), Achnanthes kuwaitensis NIES1349 (P), Asterionella glacialis NIES417 Q), Pythium ultimum DAOM BR144 (T), Spizellomyces punctatus DAOM BR117 (S), and Thecamonas trahens ATCC50062 (T)). EFL of dual-EF-containing species (Achnanthes kuwaitensis NIES1349 (U), Asterionella glacialis NIES417 (V), Spizellomyces punctatus DAOM BR117 (W) and Thecamonas trahens ATCC50062(X), Detonula confervcace CCMP353 (Y), Thalassionema nitzschioides NIES534 (Z), EFL of EFL-containing species (Goniomonas sp. NIES 1374 (A'), Raphidiophrys contractilis (AB332032) (B'), Planoglabratella opercularis (EU810334) (C'), Capromyxa protea CF08-5 (ATCC PRA-324) (D'), and Fabomonas tropica strain NYK3C (E')).

A

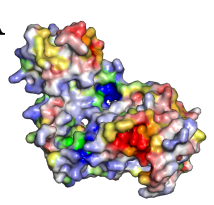

EF-1 $\alpha^{\text {single }}$

diveF-1 $\alpha^{\text {dual }}$

EFLdual

EFL single

K

P

$\mathbf{U}$
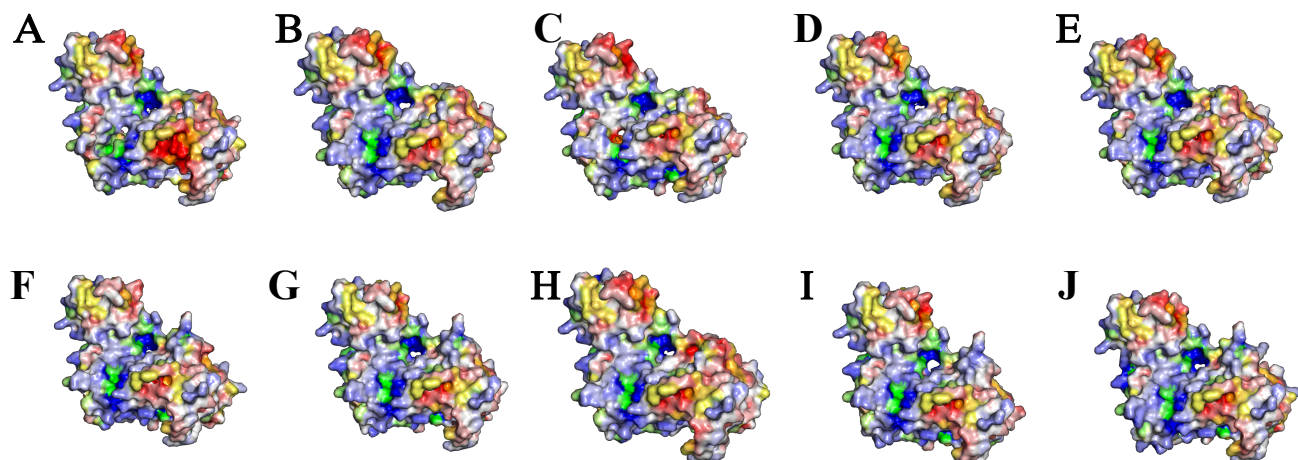

G
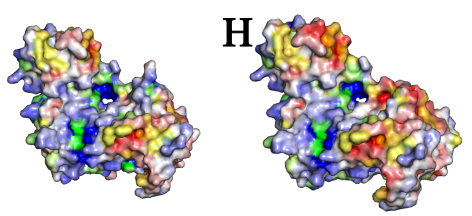

I

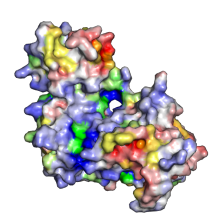

J
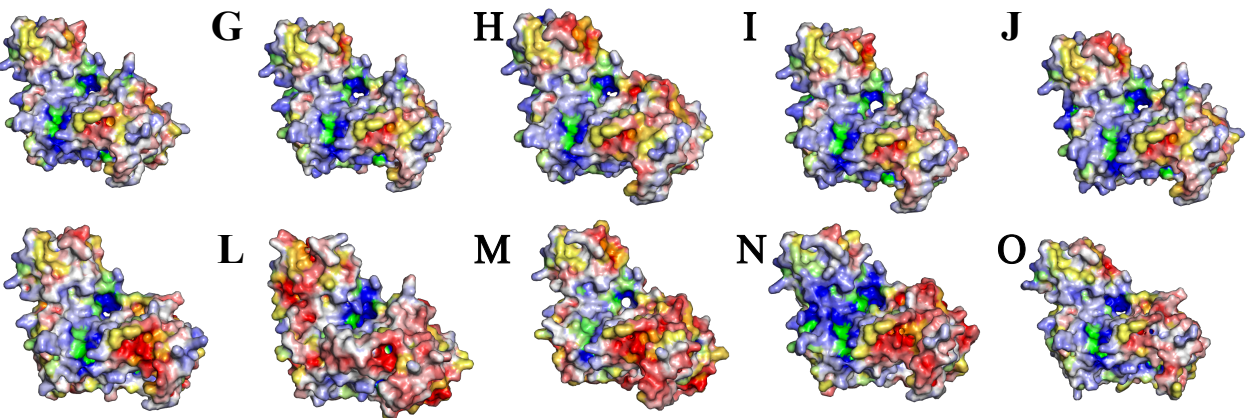

L
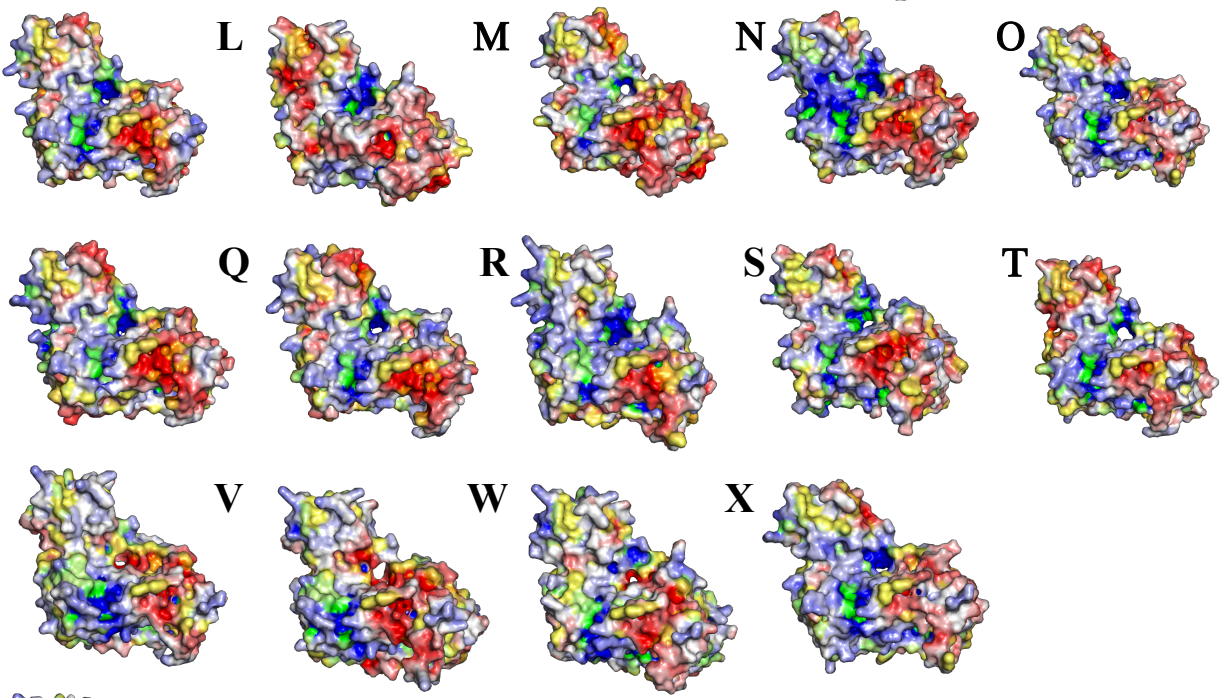
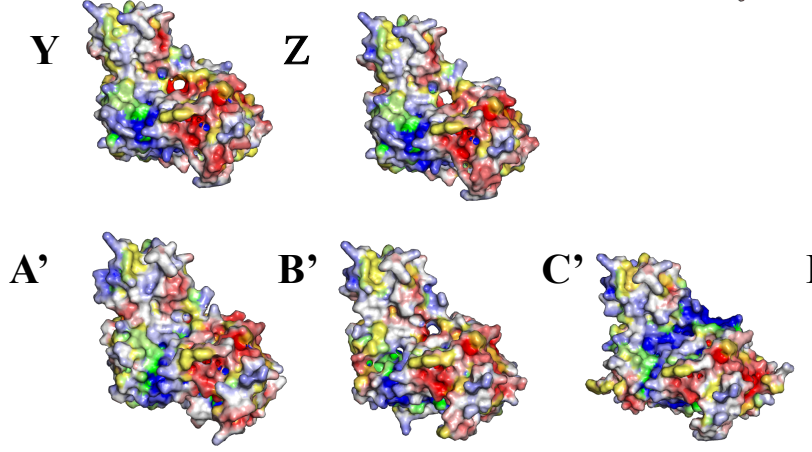

S7
D'
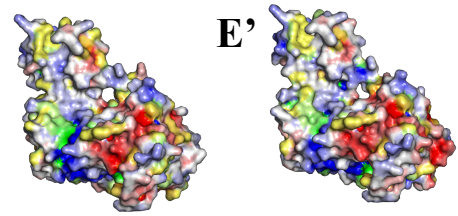

electrostatic potential
$+0.1 \mathrm{~V}$

hydrophilic hydrophobic 
Figure S4

Molecular surface seen from the tRNA-binding side of EF-1 $\alpha$ and EFL models, generated using SWISS-MODEL using 3WXMin PDB as the template, obtained using eF-surf web server [34]: EF-1 $\alpha$ of EF-1a-containing species (Bolidomonas pacifica CCMP 1866 (A), Goniomonas sp. ATCC PRA68 (B), Corallochytrium limacisporum (AY582826) (C), Goniomonas truncata NIES 1373 (D), Goniomonas sp. CCAP 980_1 (E), Subulatomonas sp. strain PCMinv5 (F), Breviata-like biflagellate strain PCbi66 (G), Roombia sp. NY0200 (H), Mantamonas plastica Bass1 (CCAP 1946/1) (I), and microaerophilic cercozoan strain DMV (J)). Divergent EF-1a of dual-EF-containing species (Detonula confervcace CCMP353 (K), Fragilariopsis cylindrus (L), Thalassionema nitzschioides NIES534 (M), Pythium intermedium MAFF306022 (N), Goniomonas sp. ATCC 50180 (O), Achnanthes kuwaitensis NIES1349 (P), Asterionella glacialis NIES417 (Q), Pythium ultimum DAOM BR144 (R), Spizellomyces punctatus DAOM BR117 (S), and Thecamonas trahens ATCC50062 (T)). EFL of dual-EF-containing species (Achnanthes kuwaitensis NIES1349 (U), Asterionella glacialis NIES417 (V), Spizellomyces punctatus DAOM BR117 (W) and Thecamonas trahens ATCC50062(X), Detonula confervcace CCMP353 (Y), Thalassionema nitzschioides NIES534 (Z), EFL of EFL-containing species (Goniomonas sp. NIES 1374 (A'), Raphidiophrys contractilis (AB332032) (B'), Planoglabratella opercularis (EU810334) (C'), Capromyxa protea CF08-5 (ATCC PRA-324) (D'), and Fabomonas tropica strain NYK3C (E')).
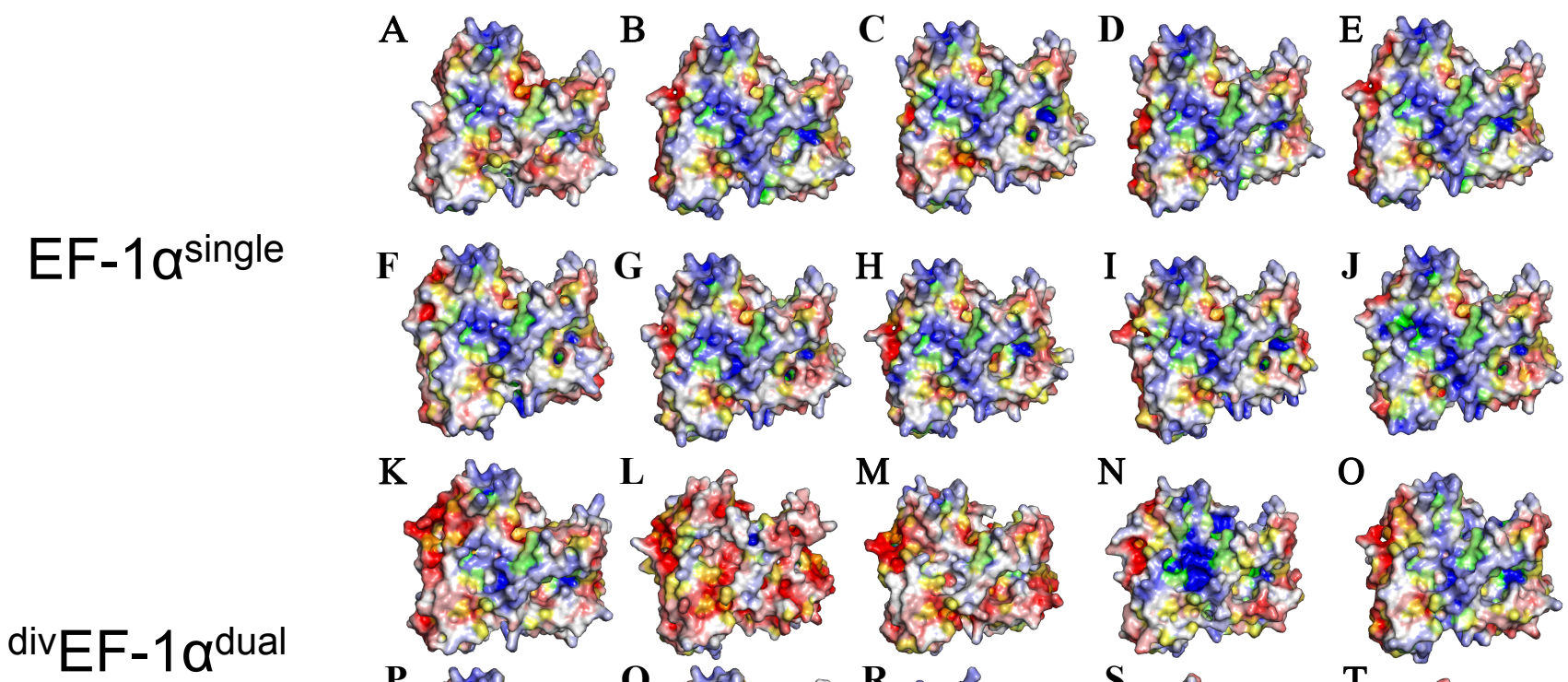

K

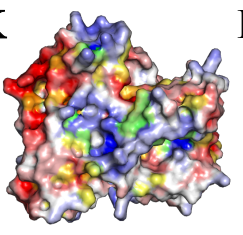

$\mathbf{P}$

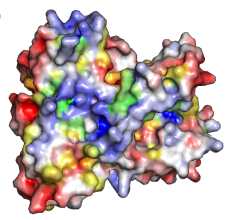

L

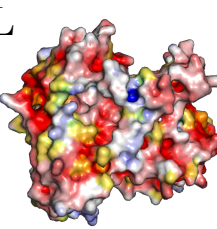

Q

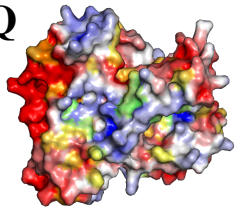

M

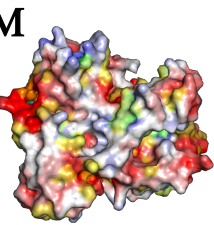

R
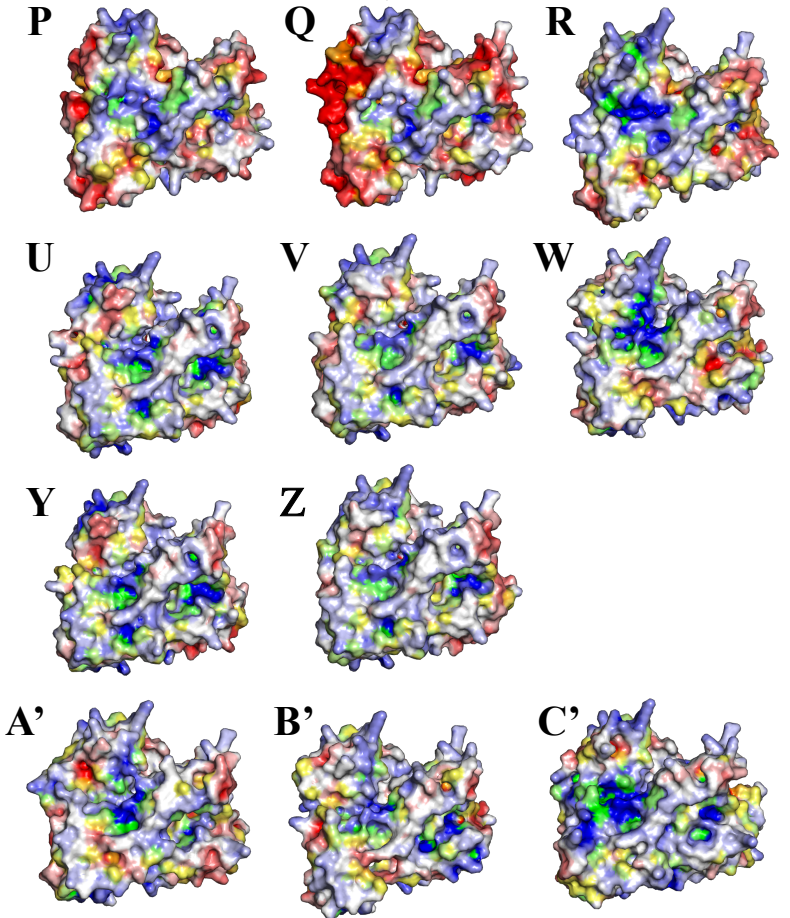
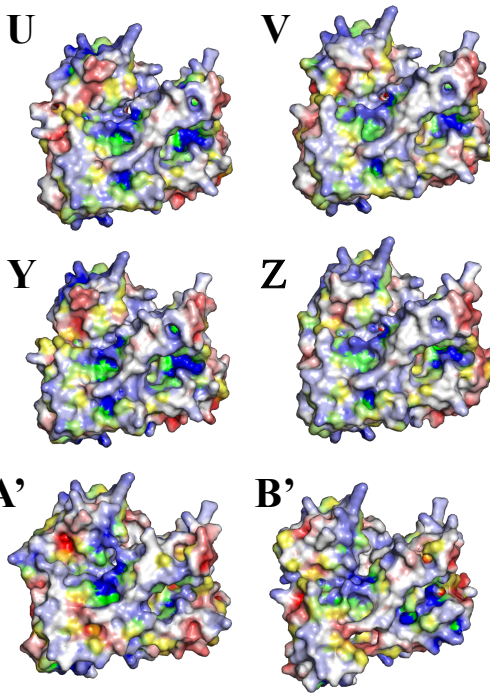

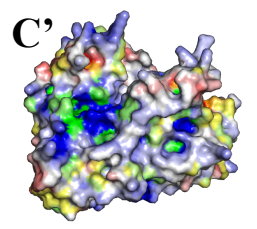

S8
N
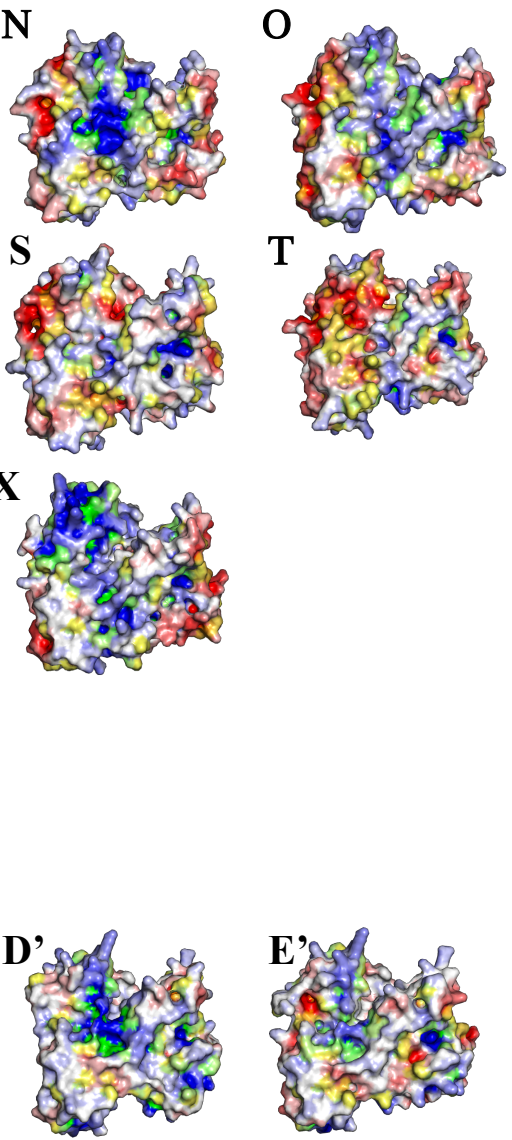

$\mathrm{T}$
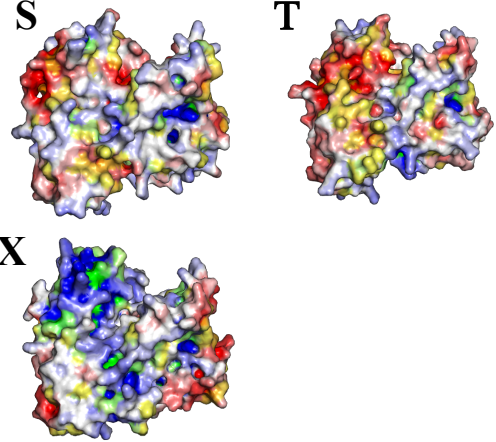

EFL single

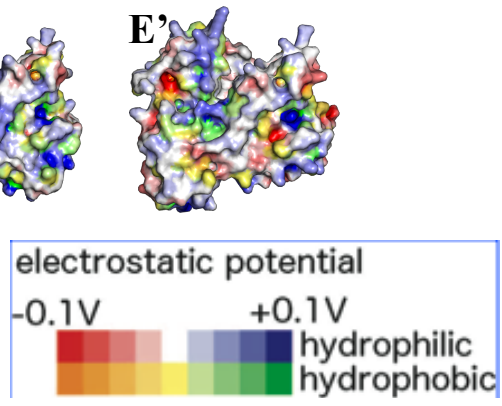


Figure S5

Molecular surface seen from the backside of EF-1 $\alpha$ and EFL models, generated using SWISS-MODEL using $3 \mathrm{WXM}$ in PDB as the template, obtained using eF-surf web server [34]: EF-1a of EF-1a-containing species (Bolidomonas pacifica CCMP 1866 (A), Goniomonas sp. ATCC PRA68 (B), Corallochytrium limacisporum (AY582826) (C), Goniomonas truncata NIES 1373 (D), Goniomonas sp. CCAP 980_1 (E), Subulatomonas sp. strain PCMinv5 (F), Breviata-like biflagellate strain PCbi66 (G), Roombia sp. NY0200 (H), Mantamonas plastica Bass1 (CCAP 1946/1) (I), and microaerophilic cercozoan strain DMV (J)). Divergent EF-1a of dual-EF-containing species (Detonula confervcace CCMP353 (K), Fragilariopsis cylindrus (L), Thalassionema nitzschioides NIES534 (M), Pythium intermedium MAFF306022 (N), Goniomonas sp. ATCC 50180 (O), Achnanthes kuwaitensis NIES1349 (P), Asterionella glacialis NIES417 (Q), Pythium ultimum DAOM BR144 (R), Spizellomyces punctatus DAOM BR117 (S), and Thecamonas trahens ATCC50062 (T)). EFL of dual-EF-containing species (Achnanthes kuwaitensis NIES1349 (U), Asterionella glacialis NIES417 (V), Spizellomyces punctatus DAOM BR117 (W) and Thecamonas trahens ATCC50062(X), Detonula confervcace CCMP353 (Y), Thalassionema nitzschioides NIES534 (Z), EFL of EFL-containing species (Goniomonas sp. NIES 1374 (A'), Raphidiophrys contractilis (AB332032) (B'), Planoglabratella opercularis (EU810334) (C'), Capromyxa protea CF08-5 (ATCC PRA-324) (D'), and Fabomonas tropica strain NYK3C (E')).

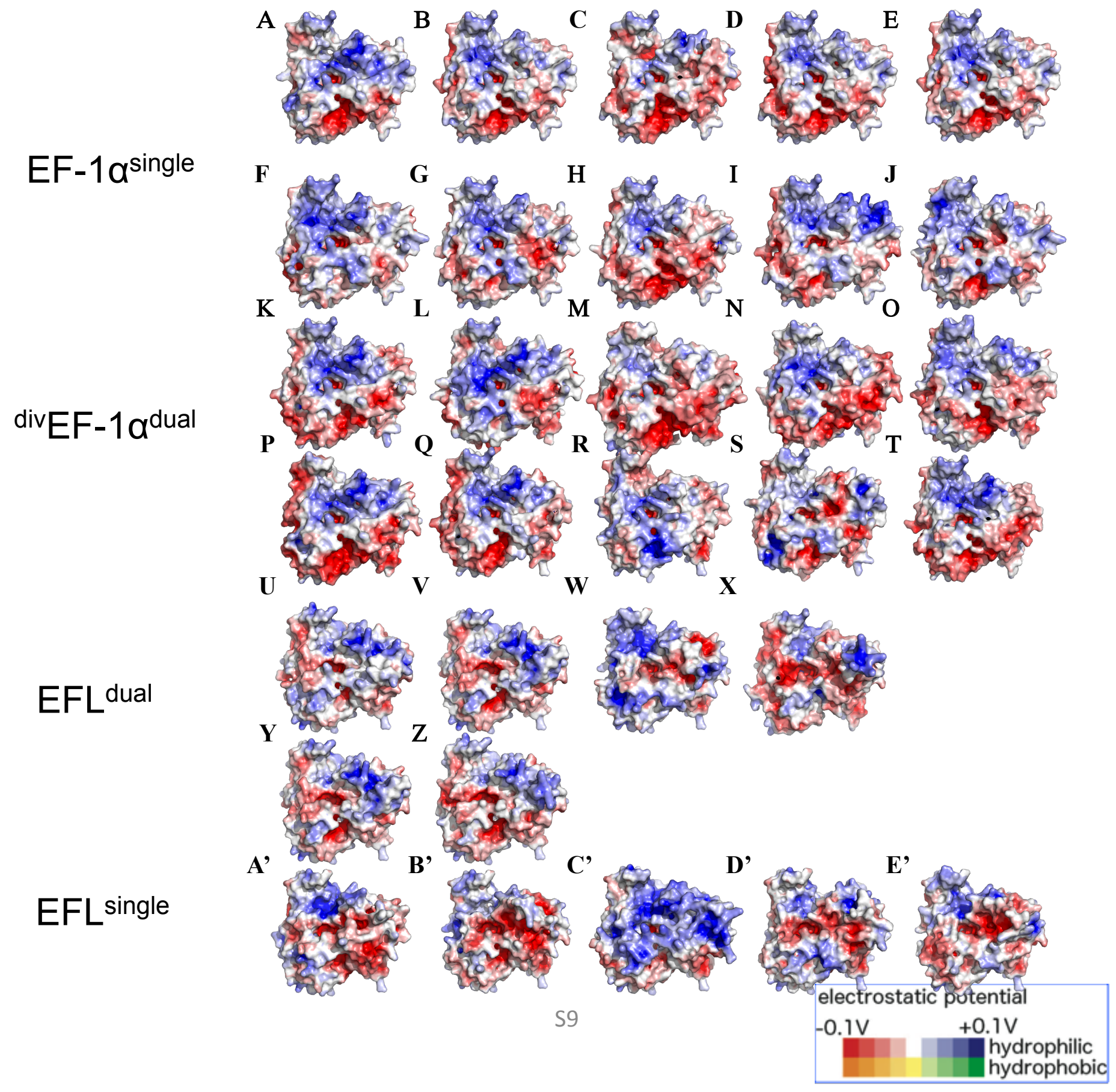


Table S5

The values of net charges and electrostatic potentials

\begin{tabular}{|c|c|c|c|c|c|}
\hline protein & taxon name & $\begin{array}{l}\text { Net } \\
\text { charge }\end{array}$ & Total & Exact & Neighbourhood \\
\hline \multirow{10}{*}{$\begin{array}{c}\text { EF-1 } \alpha \text { of } \\
\text { EF-1 } 1 \alpha \text {-containing species }\end{array}$} & Bolidomonas pacifica CCMP 1866 & $6 \mathrm{e}$ & -143.27 & -138.59 & 12.63 \\
\hline & Goniomonas sp. ATCC PRA68 & $6 \mathrm{e}$ & 1.11 & -19.39 & 73.61 \\
\hline & Corallochytrium limacisporum AY582826 & $3 \mathrm{e}$ & -550.24 & -59.41 & 42.23 \\
\hline & Goniomonas truncata NIES 1373 & $5 \mathrm{e}$ & -34.76 & -13.52 & 59.35 \\
\hline & Goniomonas sp. CCAP 980_1 & $6 \mathrm{e}$ & -20.25 & -18.78 & 63.95 \\
\hline & Subulatomonas sp. strain PCMinv5 & $9 \mathrm{e}$ & -242.68 & -22.59 & 69.35 \\
\hline & Breviata-like biflagellate strain PCbi66 & 8 e & 1.46 & -30.74 & 43.27 \\
\hline & Roombia sp. NY0200 & $5 \mathrm{e}$ & -621.08 & -50.83 & 57.88 \\
\hline & Mantamonas plastica Bass1 (CCAP 1946/1) & 8 e & 263.69 & -24.72 & 63.91 \\
\hline & microaerophilic cercozoan strain DMV & $11 \mathrm{e}$ & 3.09 & 2.09 & 110.97 \\
\hline \multirow{5}{*}{$\begin{array}{c}\text { EFL of } \\
\text { EFL-containing species }\end{array}$} & Goniomonas sp. NIES 1374 & $4 \mathrm{e}$ & 188.91 & 17.48 & 161.87 \\
\hline & Raphidiophrys contractilis AB332032 & $5 \mathrm{e}$ & -309.36 & 50.90 & 33.80 \\
\hline & Planoglabratella opercularis EU810334 & $18 \mathrm{e}$ & 2314.63 & 249.04 & 347.85 \\
\hline & Capromyxa protea CF08-5 (ATCC PRA-324) & $10 \mathrm{e}$ & 1005.38 & 141.05 & 193.52 \\
\hline & Fabomonas tropica strain NYK3C & $5 \mathrm{e}$ & 216.30 & 37.33 & 74.19 \\
\hline \multirow{6}{*}{$\begin{array}{c}\text { EFL of } \\
\text { dual-EF-containing } \\
\text { species }\end{array}$} & Achnanthes kuwaitensis NIES1349 & 8 e & 140.83 & 61.29 & 81.52 \\
\hline & Asterionella glacialis NIES417 & $7 \mathrm{e}$ & -44.35 & 12.77 & 84.09 \\
\hline & Detonula confervcace CCMP353 & $7 \mathrm{e}$ & 333.24 & 45.88 & 90.50 \\
\hline & Spizellomyces punctatus DAOM BR117 & $12 \mathrm{e}$ & 438.39 & 157.02 & 112.3 \\
\hline & Thalassionema nitzschioides NIES534 & $7 \mathrm{e}$ & 164.82 & 65.91 & 169.78 \\
\hline & Thecamonas trahens ATCC50062 & $3 \mathrm{e}$ & 642.02 & 184.58 & 259.37 \\
\hline \multirow{10}{*}{$\begin{array}{l}\text { Divergent EF-1 } \alpha \text { of } \\
\text { dual-EF-containing } \\
\text { species }\end{array}$} & Achnanthes kuwaitensis NIES1349 & $-7 \mathrm{e}$ & -524.05 & -115.56 & 47.20 \\
\hline & Asterionella glacialis NIES417 & $-11 \mathrm{e}-$ & -1029.46 & -123.70 & -74.43 \\
\hline & Detonula confervacea CCMP353 & $-2 \mathrm{e}$ & -319.42 & -70.38 & -15.82 \\
\hline & Goniomonas sp. ATCC 50180 & $1 \mathrm{e}$ & -228.22 & 21.32 & 38.27 \\
\hline & Fragilariopsis cylindrus & -10 e - & -2466.81 & -416.15 & -609.95 \\
\hline & Pythium intermedium MAFF306022 & -3 e & 83.48 & 247.74 & 275.48 \\
\hline & 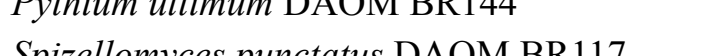 & $4 \mathrm{e}-$ & -1417.96 & -218.00 & -64.01 \\
\hline & Thalassionema nitzschioides NIES534 & $0 \mathrm{e}$ & -505.09 & -90.74 & -11.66 \\
\hline & Thecamonas trahens ATCC50062 & -13 e - & -1232.49 & -153.06 & -64.24 \\
\hline & & $-6 \mathrm{e}$ & -263.18 & -144.85 & -129.38 \\
\hline
\end{tabular}


Figure S6

The structures and molecular surfaces of the averaged structure of $100 \mathrm{~ns}$ MD simulation of Subulatomonas sp. strain PCMinv5 (A) and Pythium ultimum DAOM BR144 (B) and EFL of Thecamonas trahens ATCC50062(C) and Fabomonas tropica strain NYK3C (D).

A

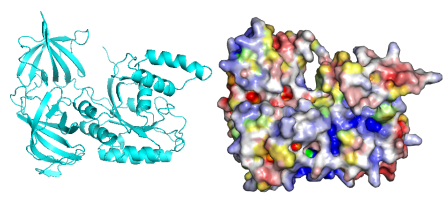

B Initial structures $\mathrm{C}$

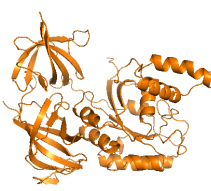

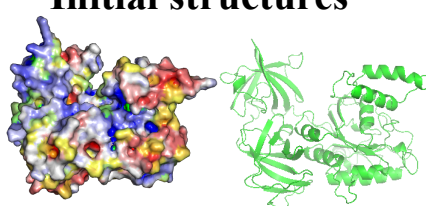

After MD

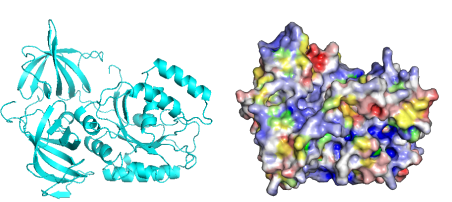

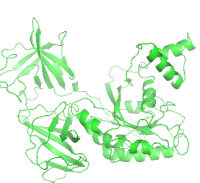

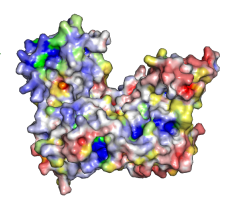

$\mathrm{D}$
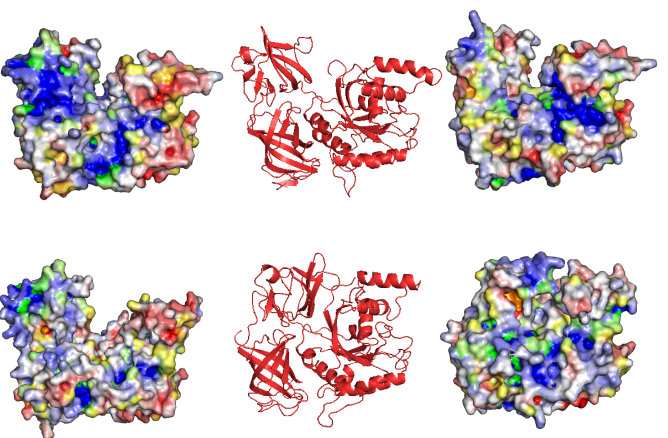
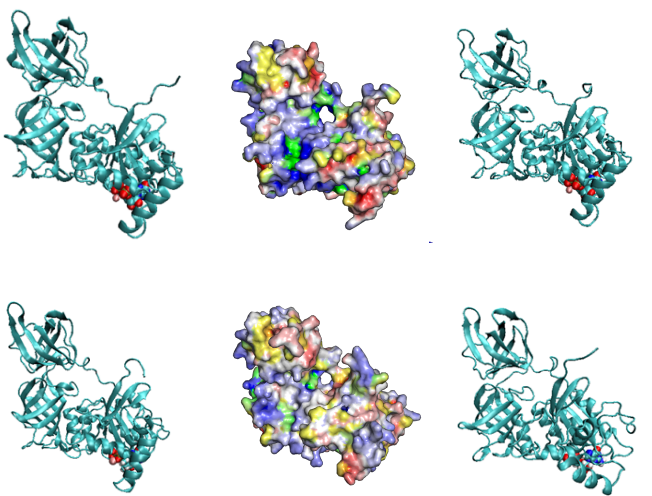
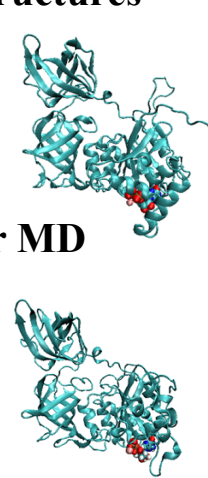
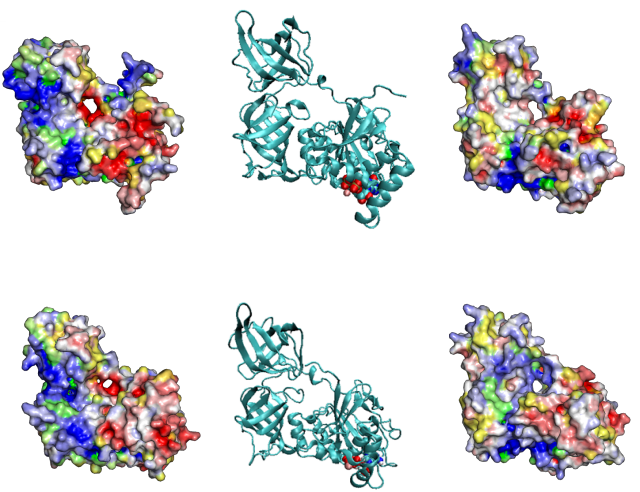

\section{Initial structures}
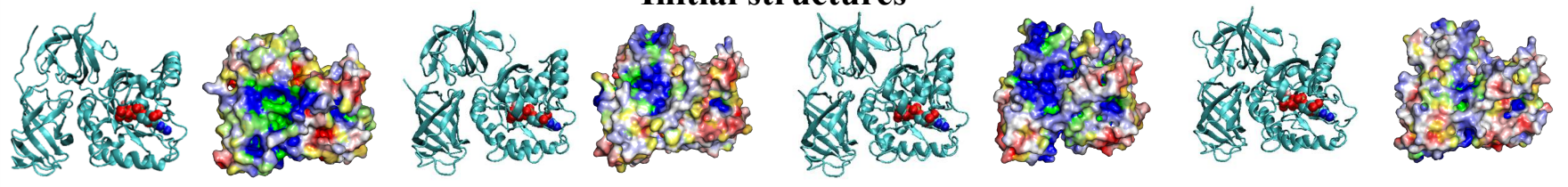

After MD
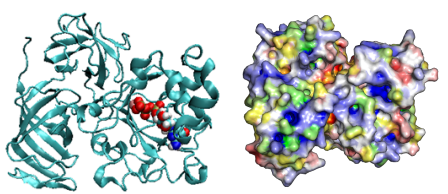
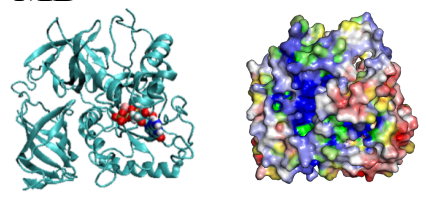

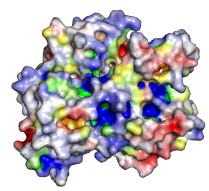




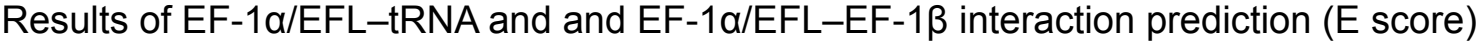

\begin{tabular}{|c|c|c|c|c|c|c|c|}
\hline \multirow[t]{2}{*}{ protein } & \multirow[t]{2}{*}{ taxon name } & \multicolumn{3}{|c|}{ phe-tRNA } & \multicolumn{3}{|c|}{ EF-1及 } \\
\hline & & $1 \mathrm{G} 7 \mathrm{C}$ & $4 \mathrm{C} 0 \mathrm{~S}$ & $3 \mathrm{WXM}$ & $1 \mathrm{G} 7 \mathrm{C}$ & $4 \mathrm{C} 0 \mathrm{~S}$ & $3 \mathrm{WXM}$ \\
\hline & templates & 9.83 & 6.96 & 5.10 & 20.83 & 8.48 & 5.21 \\
\hline \multirow{11}{*}{$\begin{array}{c}\text { EF-1 } \alpha \text { of } \\
\text { EF-1 } \alpha \text {-containing } \\
\text { species }\end{array}$} & Bolidomonas pacifica CCMP 1866 & 10.73 & 6.92 & 7.32 & 20.83 & 6.05 & 5.26 \\
\hline & Goniomonas sp. ATCC PRA68 & 8.35 & 6.76 & 9.37 & 19.34 & 5.06 & 6.63 \\
\hline & Corallochytrium limacisporum AY582826 & & & & & & \\
\hline & Goniomonas truncata NIES 1373 & 10.19 & 0.07 & 11.03 & 11.01 & 3.19 & 3.91 \\
\hline & & 6.83 & 6.76 & 7.37 & 17.47 & 5.09 & 6.60 \\
\hline & 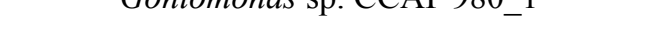 & 8.85 & 6.48 & 9.33 & 17.50 & 5.93 & 6.64 \\
\hline & Subulatomonas sp. strain PCMinv5 & 5.35 & 7.48 & 9.05 & 17.94 & 8.17 & 5.52 \\
\hline & Breviata-like biflagellate strain PCbi66 & 9.96 & 5.43 & 10.13 & 19.62 & 7.45 & 7.31 \\
\hline & Roombia sp. NY0200 & 10.77 & 6.23 & 8.30 & 18.73 & 6.02 & 6.77 \\
\hline & Mantamonas plastica Bass1 (CCAP 1946/1) & 9.19 & 6.32 & 9.36 & 18.23 & 7.63 & 5.99 \\
\hline & microaerophilic cercozoan strain DMV & 5.75 & 6.48 & 11.54 & 17.61 & 9.09 & 5.20 \\
\hline \multirow{10}{*}{$\begin{array}{c}\text { Divergent EF-1 } \alpha \text { of } \\
\text { dual-EF-containing } \\
\text { species }\end{array}$} & Detonula confervcace CCMP353 & 5.55 & 6.22 & 8.60 & 16.64 & 6.27 & 5.71 \\
\hline & Fragilariopsis cylindrus & 7.06 & 7.08 & 5.89 & 13.32 & 6.67 & 5.64 \\
\hline & Goniomonas sp. ATCC 50180 & 10.99 & 7.80 & 10.73 & 16.46 & 6.10 & 5.28 \\
\hline & Pythium intermedium MAFF306022 & 7.57 & 6.80 & 6.40 & 12.21 & 5.38 & 5.52 \\
\hline & Thalassionema nitzschioides NIES534 & 10.29 & 6.16 & 7.82 & 17.60 & 6.04 & 6.03 \\
\hline & Achnanthes kuwaitensis NIES1349 & 6.09 & 5.92 & 8.73 & 20.55 & 7.61 & 6.61 \\
\hline & Asterionella glacialis NIES417 & 8.29 & 6.69 & 8.97 & 16.18 & 5.30 & 6.77 \\
\hline & Pythium ultimum DAOMBR144 & 6.25 & 6.49 & 5.97 & 17.56 & 6.87 & 7.13 \\
\hline & Spizellomyces punctatus DAOMBR117 & 10.28 & 10.50 & 6.56 & 21.88 & 8.40 & 4.73 \\
\hline & Thecamonas trahens ATCC50062 & 5.55 & 8.44 & 5.53 & 16.90 & 6.27 & 6.67 \\
\hline \multirow{6}{*}{$\begin{array}{c}\text { EFL of } \\
\text { dual-EF-containing } \\
\text { species }\end{array}$} & Achnanthes kuwaitensis NIES1349 & 6.22 & 5.98 & 6.20 & 19.60 & 5.23 & 6.24 \\
\hline & Asterionella glacialis NIES417 & 5.83 & 6.04 & 7.39 & 16.89 & 5.74 & 5.59 \\
\hline & Spizellomyces punctatus DAOMBR117 & 6.43 & 5.70 & 6.21 & 18.30 & 5.53 & 6.09 \\
\hline & Thecamonas trahens ATCC50062 & 7.84 & 5.18 & 6.77 & 12.54 & 6.82 & 6.56 \\
\hline & Thalassionema nitzchioides NIES534 & 4.78 & 5.72 & 6.20 & 18.90 & 6.06 & 6.03 \\
\hline & Detonula confervcace CCMP353 & 6.90 & 6.07 & 7.39 & 17.27 & 5.65 & 5.71 \\
\hline \multirow{5}{*}{$\begin{array}{c}\text { EFL of } \\
\text { EFL-containing } \\
\text { species }\end{array}$} & Goniomonas sp. NIES 1374 & 5.86 & 5.72 & 6.41 & 17.18 & 5.61 & 5.58 \\
\hline & AB368774 & 5.63 & 6.07 & 5.02 & 18.57 & 6.57 & 4.76 \\
\hline & EU810334 & 5.50 & 5.72 & 5.97 & 19.02 & 5.70 & 6.18 \\
\hline & Capromyxa protea CF08-5 (ATCC PRA-324) & & & & & & \\
\hline & Fabomonas tropica strain NYK3C & $\begin{array}{l}6.11 \\
6.67\end{array}$ & $\begin{array}{l}0.01 \\
5.72\end{array}$ & $\begin{array}{l}5.54 \\
5.75\end{array}$ & $\begin{array}{l}15.8 / \\
17.39\end{array}$ & $\begin{array}{l}4.98 \\
6.21\end{array}$ & $\begin{array}{l}5.81 \\
5.03\end{array}$ \\
\hline
\end{tabular}




\section{Table S7}

Results of EF-1 $\alpha /$ EFL-tRNA interaction prediction ( $E$ score)

\begin{tabular}{|c|c|c|}
\hline protein & taxon name & E score \\
\hline \multirow{6}{*}{$\begin{array}{c}\text { EF-1 } \alpha \text { of } \\
\text { EF-1 } \alpha \text {-containing } \\
\text { species }\end{array}$} & Subulatomonas sp. strain PCMinv5 & 5.68 \\
\hline & Subulatomonas sp. strain PCMinv5 afterMD & 5.54 \\
\hline & Subulatomonas sp. strain PCMinv5(GDP) & 8.06 \\
\hline & Subulatomonas sp. strain PCMinv5(GDP) after MD & 6.00 \\
\hline & Subulatomonas sp. strain PCMinv5(GTP) & 7.43 \\
\hline & Subulatomonas sp. strain PCMinv5(GTP) after MD & 5.35 \\
\hline \multirow{6}{*}{$\begin{array}{c}\text { EFL of } \\
\text { EFL-containing } \\
\text { species }\end{array}$} & Fabomonas tropica strain NYK3C & 6.95 \\
\hline & Fabomonas tropica NYK3C (afterMD) & 4.83 \\
\hline & Fabomonas tropica NYK3C(GDP) & 6.70 \\
\hline & Fabomonas tropica NYK3C(GDP) after MD & 5.98 \\
\hline & Fabomonas tropica NYK3C(GTP) & 6.66 \\
\hline & Fabomonas tropica NYK3C(GTP) after MD & 6.31 \\
\hline \multirow{6}{*}{$\begin{array}{c}\text { Divergent EF-1 } \alpha \text { of } \\
\text { dual-EF-containing } \\
\text { species }\end{array}$} & Thecamonas trahens ATCC50062 & 5.90 \\
\hline & Thecamonas trahens ATCC50062(afterMD) & 6.28 \\
\hline & Thecamonas trahens ATCC50062(GDP) & 5.34 \\
\hline & Thecamonas trahens ATCC50062(GDP) after MD & 5.42 \\
\hline & Thecamonas trahens ATCC50062(GTP) & 6.72 \\
\hline & Thecamonas trahens ATCC50062(GTP) after MD & 7.09 \\
\hline \multirow{6}{*}{$\begin{array}{c}\text { Divergent EF-1 } \alpha \text { of } \\
\text { dual-EF-containing } \\
\text { species }\end{array}$} & Pythium ultimum DAOMBR144 & 7.04 \\
\hline & Pythium ultimum DAOMBR144(afterMD) & 4.98 \\
\hline & Pythium ultimum DAOMBR144(GDP) & 7.01 \\
\hline & Pythium ultimum DAOMBR144(GDP) after MD & 7.03 \\
\hline & Pythium ultimum DAOMBR144(GTP) & 6.44 \\
\hline & Pythium ultimum DAOMBR144(GTP) after MD & 8.24 \\
\hline
\end{tabular}

\section{$E$ value for phetRNA (1EHZ)}

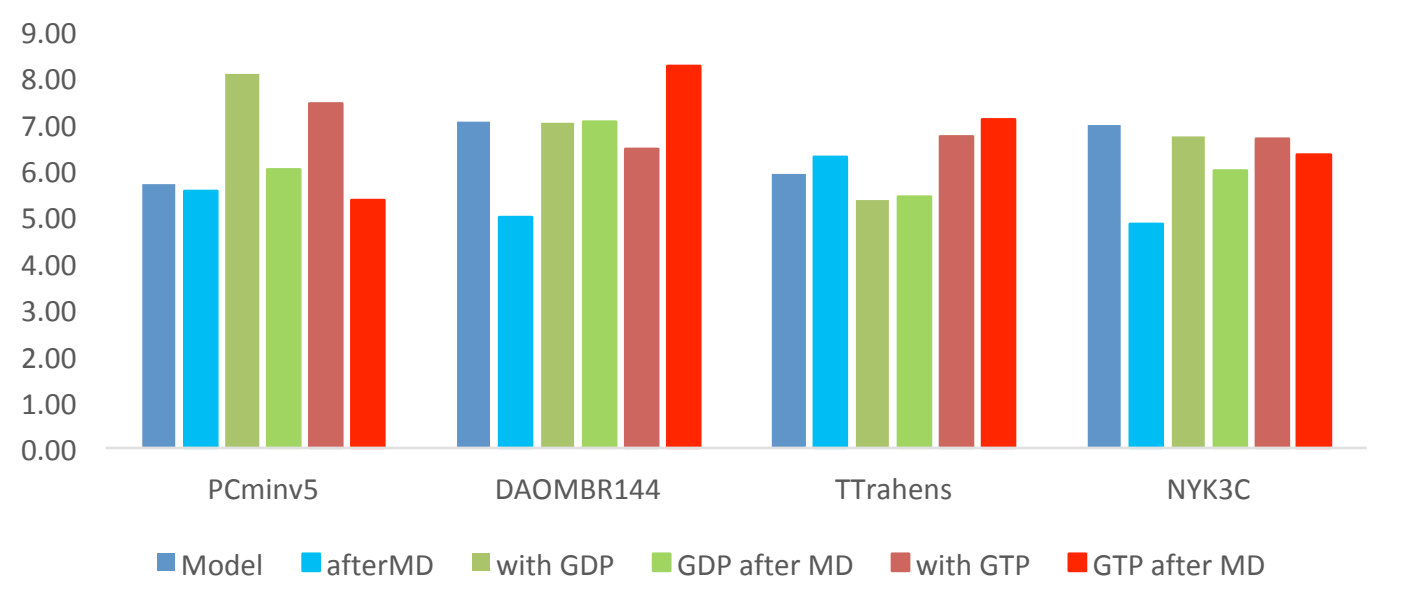

